Preprints of the

Max Planck Institute for

Research on Collective Goods

Bonn 2009/37

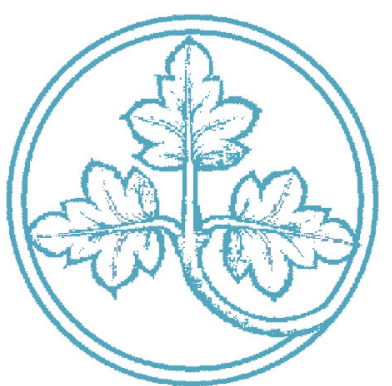

Das schwindende Vertrauen in die Marktwirtschaft und die Folgen für das Recht

Christoph Engel

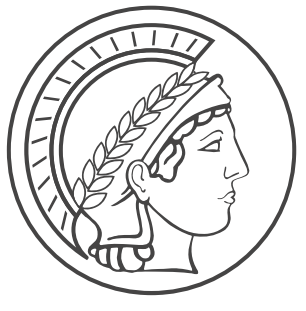




\title{
Das schwindende Vertrauen in die Marktwirtschaft und die Folgen für das Recht*
}

\author{
Christoph Engel
}

October 2009

* Felix Bierbrauer, Martin Hellwig und Jörn Lüdemann danke ich für hilfreiche Hinweise und Kritik zu einer früheren Fassung. 


\section{Grund zur Sorge, aber auch Grund zum Handeln?}

Nichts ist so wirksam wie das Selbstverständliche. Deutschland ist eine Marktwirtschaft. Über Jahrzehnte war das für die meisten Deutschen nur die Feststellung einer Tatsache, keine normative Aussage. In den Geschichtsbüchern stand, dass man es auch anders machen könnte. Aber die praktischen Beispiele wirkten so wenig einladend, dass so recht niemand Neigung verspürte, die Wirtschaftsordnung als solche in Frage zu stellen. Man hatte sich eingerichtet und forderte lieber mehr Einkommen oder niedrigere Steuern.

Das hat sich mehr schleichend als eruptionsartig geändert. Demoskopische Untersuchungen legen nahe, dass der Großteil der Deutschen die Entscheidung für die Marktwirtschaft nicht mehr für fraglos hält (2). Soll die Rechtsordnung darauf reagieren? Darf sie das? Dagegen kann man zunächst einen rechtstheoretischen Einwand erheben: Die Aufgabe des Rechts ist die Ordnung des Sollens, nicht die Verarbeitung des Seins (3). Auch wer so radikal nicht sein will, kann sich als Verfassungsrechtler doch fragen, ob die Rechtsordnung Eingriffe in Freiheit und Eigentum auf eine so weiche Grundlage wie die Einstellungen der Bürger stellen darf (4). Dieser Aufsatz möchte das nicht kategorisch ausschließen. Der Zusammenhang zwischen weit verbreiteten Einstellungen und Handlungen, die die Rechtsordnung problematisch finden könnte, ist aber ziemlich vermittelt. Kompensierend muss das normative Anliegen hinreichendes Gewicht haben (5). Es ergibt sich hier aus der fundamentalen Bedeutung von generalisiertem Vertrauen (6). Damit sind die Maßstäbe entwickelt, um eine Entscheidung verfassungsrechtlich zu beurteilen, über die intensiv diskutiert wird: eine Begrenzung der Managergehälter (7). Sie scheinen als Signal für das Ausmaß solidarischer Gleichheit zu wirken, zu dem die Wirtschaftsordnung führt. Für diese Aussage gibt es im Moment zwar keinen direkten Nachweis. Immerhin gibt es aber ein deutliches Indiz: das Urteil der Befragten über die Gerechtigkeit der Marktwirtschaft lässt sich mit dem Maß der objektiven Ungleichheit der Einkommen erklären (8).

\section{Das schwindende Vertrauen in die Marktwirtschaft}

Der Gegenstand dieses Aufsatzes ist die Einstellung der Deutschen zur Marktwirtschaft. Wie soll man einen so weichen Gegenstand in harte Zahlen übersetzen? Das Institut für Demoskopie in Allensbach hat einen Weg gefunden. Nach der Wiedervereinigung wollte es wissen, wie die neuen Bundesbürger zu der ganz anderen Wirtschaftsordnung im Westen stehen ${ }^{1}$. Sie waren begeistert. Schon ein Jahr später hatte aber nur noch gut die Hälfte der Befragten eine gute Meinung von Marktwirtschaft. Ab 1992 liegt die Zahl konstant unter 50\%. Im Jahre 2005 waren es gerade noch 14\%. Wie Abbildung 1 zeigt, sieht es im Westen nur wenig besser aus.

1 Die Frage lautete: „Haben Sie vom Wirtschaftssystem in Deutschland eine gute Meinung oder keine gute Meinung?“. 


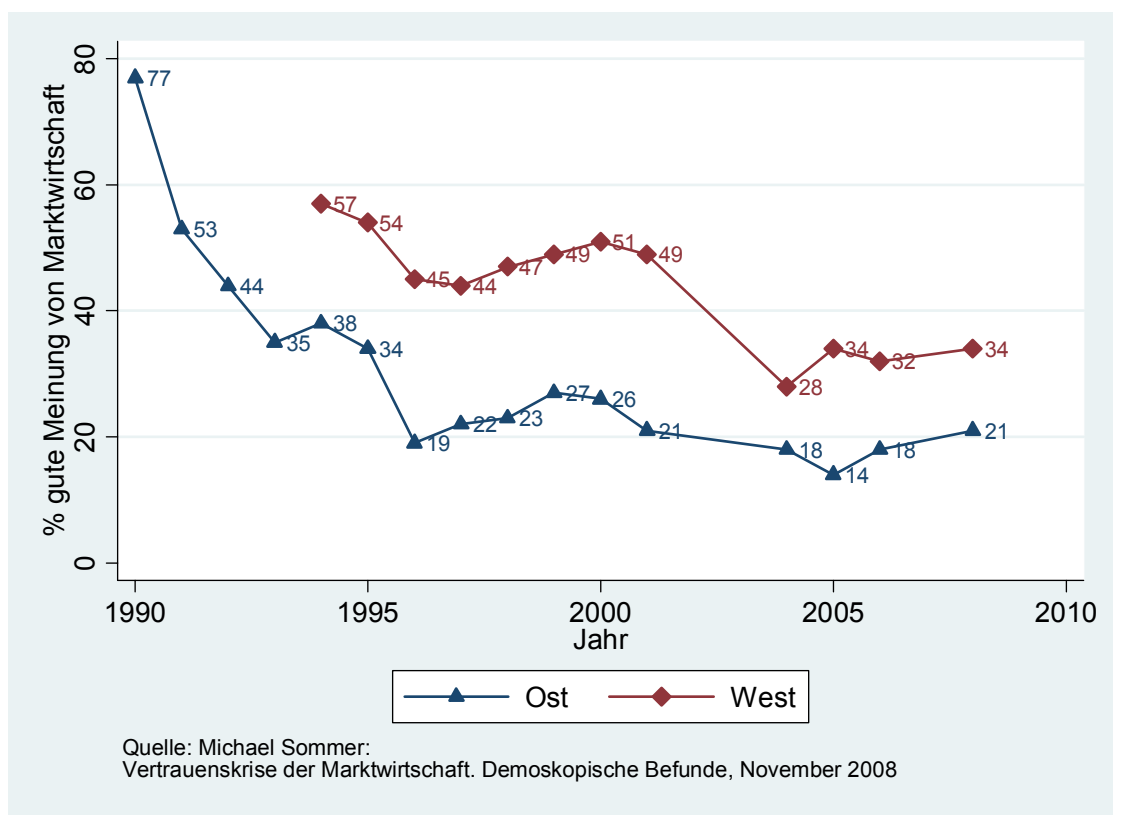

Abbildung 1

Einstellung zur Marktwirtschaft

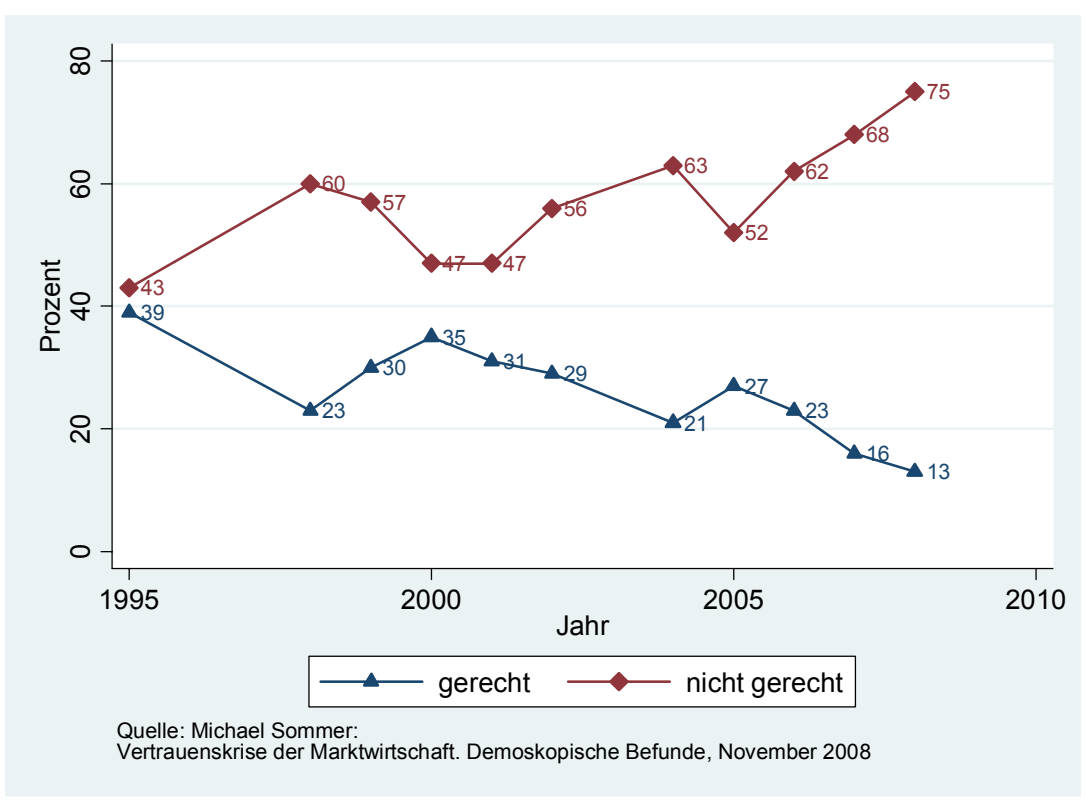

Abbildung 2

Bewertung der Gerechtigkeit der Wirtschaftsordnung

Die Ablehnung ist noch eindeutiger, wenn man die Menschen fragt, ob sie die Marktwirtschaft für gerecht halten ${ }^{2}$. Seit 1995 antworten mehr Menschen mit Nein als mit Ja. Anfangs lagen beide Antworten aber noch nahe beieinander. Seither klafft die Schere immer weiter auseinander.

2 Die Frage lautete: „Wie sehen Sie das: sind die wirtschaftlichen Verhältnisse bei uns in Deutschland - ich meine, was die Menschen besitzen und was sie verdienen - im Großen und Ganzen gerecht oder nicht gerecht?". 
Im Jahre 2008 hielten nur noch 13\% der Befragten die Marktwirtschaft für gerecht, während $75 \%$ sie eindeutig für ungerecht hielten.

\section{Recht und Wirklichkeit}

Wenn die Tatsachen gegen mich sind, dann desto schlimmer für die Tatsachen! Dieser trotzige Ausruf wird Georg Wilhelm Friedrich Hegel zugeschrieben. Ein Jurist braucht für diese Haltung gar keinen philosophischen Kronzeugen. Es genügt, wenn er sich in der Rechtstheorie auf die Seite derer schlägt, denen Konsistenz über alles geht. So berühmte Autoren wie Ronald Dworkin werben dafür, Rechtsregeln nicht nach ihrer Kraft zur Bewältigung sozialer Probleme zu beurteilen. Stattdessen soll eine Rechtsregel dann gut sein, wenn sie sich widerspruchsfrei in die Rechtsordnung einfügt ${ }^{3}$. Als Wahrheitskriterium kommt es dann auf Kohärenz an, nicht auf Korrespondenz (zur Wirklichkeit) ${ }^{4}$.

Wer so denkt, der wird die Allensbacher Zahlen von vornherein für unerheblich halten. Er mag um des Arguments willen zugestehen, dass sich die Wirklichkeit oder ihre Wahrnehmung geändert hat. Er mag bereit sein einzuräumen, dass die Durchsetzung des Rechts dadurch schwieriger geworden ist. Aber das sind Aufgaben für die Polizei, für die Gerichte und notfalls für die Gefängnisverwaltung. Am normativen Anspruch des Rechts ändert sich dadurch gar nichts. Im Grunde muss solch ein Jurist sogar sagen: im Gegenteil; erst dann, wenn die Befolgung des Rechts nicht mehr selbstverständlich ist, bewährt sich sein normativer Anspruch. In einem vordergründigen Sinne ist das Recht ja genau deshalb von der souveränen Allgewalt des Staats gestützt. In einem tieferen Sinne sind Normen dadurch definiert, dass sie potentiell kontrafaktisch wirken.

Diese Position hat eine klare normative Implikation. Die Tatsachen sind das Wirkungsfeld des Rechts; in seiner Auslegung haben sie nichts zu suchen. Wenn die Tatsachen der normativen Erwartung einer Rechtsnorm widersprechen, besteht Anlass zur Rechtsdurchsetzung, nicht zur Veränderung des Rechts. Auch die Gewaltenteilung zwischen dem Parlament einerseits und der Verwaltung und den Gerichten andererseits ist dann eindeutig. Das Parlament soll sich in die Regelanwendung nicht einmischen. Verwaltung und Gerichte haben kein Mandat zur Regelbildung. Wenn die fortbestehende Rechtsregel im Lichte veränderter Verhältnisse ungerecht erscheint, mag der Gesetzgeber eingreifen. Solange er sich dazu nicht entschließen kann, setzen Exekutive und Judikative die Regel durch.

Man sollte diese Position nicht verächtlich machen. Rechtsregeln werden gerade deshalb gebraucht, weil man über normative Urteile streiten kann. Denn solche Urteile beruhen auf normativen Prämissen. Es ist nicht schwer zu zeigen, dass man über ein und dieselbe normative Frage

3 R. M. Dworkin, Taking Rights Seriously. Cambridge (1977); R. M. Dworkin, Law's Empire. Cambridge, Mass. (1986).

4 Zum Hintergrund Christoph Engel, Inconsistency in the Law. In Search of a Balanced Norm, in: Daston und Engel (Hrsg.), Is There Value in Inconsistency? Baden-Baden (2006) 221-281. 
ganz unterschiedlich urteilen kann, je nachdem, welchen normativen Ausgangspunkt man wählt ${ }^{5}$. Höchste Effizienz kann zum Beispiel im Widerspruch zu größter Freiheit stehen ${ }^{6}$. Eine Urnorm, aus der sich alle normativen Urteile ableiten ließen, gibt es nicht ${ }^{7}$. Deshalb sind normative Urteile unvermeidlich politisch. Weil jeder Abgeordnete bei der nächsten Wahl sein Mandat riskiert, ist das Parlament zu offen politischen Entscheidungen legitimiert. Verwaltung und Gerichte sind es nicht. Außerdem soll das Recht gerade diejenigen schützen, die sich ohne Recht schlecht selbst schützen könnten. Wenn sich das Recht zu leicht an veränderte Tatsachen anpasst, kann es zum Machtmittel derer mutieren, die ohnehin schon im Vorteil waren.

Dieser Aufsatz schlägt sich trotzdem auf die andere Seite. Er ist von der Überzeugung getragen, dass die Rechtsordnung gravierende Veränderungen der Wirklichkeit weder ignorieren kann noch ignorieren sollte. Erscheinen erhebliche Anpassungen angezeigt, sollte nach Möglichkeit natürlich der Gesetzgeber aktiv werden. Das alte Recht muss schon schreiend ungerecht geworden sein, damit die Gerichte eingreifen können. Ein anschauliches Beispiel ist die Aufwertungsrechtsprechung des Reichsgerichts. Durch die Hyperinflation nach dem Ersten Weltkrieg waren Ansprüche auf Geld praktisch wertlos geworden. Weil das gut organisierten Gruppen nützte, die im Reichstag wirksam vertreten waren, scheiterten alle Versuche zur Milderung der alten Regel „Mark ist Mark“. In einem Befreiungsschlag hat sich das Reichsgericht schließlich zur Anpassung der Verträge durchgerungen ${ }^{8}$.

Im Alltag der Rechtsanwendung geht es meist aber auch gar nicht um den Konflikt mit dem Gesetzgeber. Oft arbeitet der Gesetzgeber sehenden Auges mit Generalklauseln, oder er gibt der Verwaltung ein Ermessen. Recht besteht aus gutem Grund aus allgemeinen Regeln. Dann muss der Rechtsanwender das Programm des Gesetzes im Lichte der Einzelheiten des Falles erst entfalten. Auch wenn die Regeln scheinbar konkret sind, muss die Anwendung der einen Regel doch in das Geflecht vieler anderer Regeln eingepasst werden. In den meisten Fällen entstehen dadurch erhebliche Auslegungsspielräume, die nur die Verwaltung und die Gerichte füllen können. Ändert sich die Wirklichkeit gravierend, auf die diese Regeln einwirken wollen, dann sollte das Rückwirkungen darauf haben, wie die Exekutive und die Judikative die Auslegungsspielräume nutzen.

Eindringlich Michael Thompson, Richard Ellis und Aaron B. Wildavsky, Cultural Theory. Boulder, Colo. (1990).

6 Das ist der Kern des Konflikts zwischen den Ordoliberalen und den Industrieökonomen, s. Martin Hellwig, Effizienz oder Wettbewerbsfreiheit? Zur normativen Grundlegung der Wettbewerbspolitik, in: Engel und Möschel (Hrsg.), Recht und spontane Ordnung. Festschrift für Ernst-Joachim Mestmäcker zum achtzigsten Geburtstag. Baden-Baden (2006) 231-268.

7 Näher Christoph Engel, Offene Gemeinwohldefinitionen, in: Rechtstheorie 32 (2001) 23-52.

8 RG 28.11.1923, RGZ 107, 78; zum Hintergrund anschaulich Knut Wolfgang Nörr, Zwischen den Mühlsteinen. Eine Privatrechtsgeschichte der Weimarer Republik. Tübingen (1988),55-71. 


\section{Veränderung der Einstellung zur Marktwirtschaft als legitimes Ziel}

Souveräne Befugnisse sind nicht nur eine Lösung. Sie sind auch eine Gefahr. Der Souverän kann seine Befugnisse missbrauchen. Benevolente Diktatoren sind selten. Herrscher können selbst dann irren, wenn sie guten Willens sind. Der Verfassungsstaat hat auf diese historischen Erfahrungen reagiert. Er hat die Rechtsordnung mehrstufig gemacht. Der Gesetzgeber übt zwar die souveräne Macht zur Regelbildung aus. Diese Macht ist ihm aber nur in den Grenzen der Verfassung anvertraut. Über die Einhaltung dieser Grenzen wacht das Verfassungsgericht. Dürfte der Gesetzgeber auf die Allensbacher Zahlen also mit Eingriffen in Freiheit und Eigentum reagieren? Dürfte er zum Beispiel eine Obergrenze für die Gehälter von Managern vorschreiben, wenn er überzeugt ist, dass hier ein Grund für die Erosion der Zustimmung zur Marktwirtschaft liegt?

In Deutschland zieht die Verfassung dem Gesetzgeber nicht nur formale Grenzen. Es genügt nicht, dass der Gesetzgeber eine Kompetenz hat und dass er die Regeln über das Gesetzgebungsverfahren beachtet. Das neue Gesetz muss auch materiell mit der Verfassung vereinbar sein. Unbedingte Verbote enthält die Verfassung allerdings nur ganz ausnahmsweise. Ein Beispiel ist das Verbot der Todesstrafe in Art. 102 GG. Sehr viel umfangreicher ist der Katalog bedingter Verbote. Genau genommen ist er durch die Interpretation von Art. 2 I GG als allgemeine Handlungsfreiheit ${ }^{9}$ sogar umfassend. In den Schutzbereich der Grundrechte darf der Gesetzgeber eingreifen, wenn er dabei den Verhältnismäßigkeitsgrundsatz beachtet. Die Maßstäbe der Geeignetheit, Erforderlichkeit und Angemessenheit stehen zwar im Mittelpunkt jeder Grundrechtsprüfung. Sie sind aber sämtlich relativ. Welche Kraft sie haben, hängt davon ab, welche normativen Ziele der Gesetzgeber vorfolgen darf. Gäbe die Verfassung ihm bei der Wahl des Ziels carte blanche, würde der Verhältnismäßigkeitsgrundsatz wertlos. Findige Juristen würden immer ein Ziel nennen können, das den Eingriff verhältnismäßig macht. Zu rigoros dürfen die Verfassungsrechtler den Katalog zulässiger normativer Ziele aber auch nicht formulieren. Sonst ersetzt man schließlich die Diktatur der gewählten Repräsentanten durch eine Diktatur der Richter.

Über die Vorgaben der Verfassung für die Bestimmung und Begrenzung legitimer Ziele ist erstaunlich wenig nachgedacht worden ${ }^{10}$. Die Frage hat jedenfalls zwei Aspekte: welche normativen Anliegen kommen überhaupt in Betracht? Wie gut muss sich nachweisen lassen, dass das vorgebrachte normative Anliegen ohne eine Intervention des Gesetzgebers tatsächlich gefährdet wäre? Beide Fragen stehen in einem inneren Zusammenhang. Wenn zu besorgen ist, dass andernfalls ein Mensch stirbt, eine Naturkatastrophe ausbricht oder eine Revolte unkontrollierbar wird, dann muss der Staat schon reagieren können, wenn ein plausibler Verdacht besteht. Das

9 BVerfGE 80, 137 - Reiten im Walde; in den letzten Jahren gibt es allerdings die bekannte Tendenz, einzelne Verhaltensweisen ganz aus dem Schutzbereich aller Grundrechte auszunehmen, BVerfGE 105, 252 - Glykol; BVerfGE 105, 279 - Osho; zurecht kritisch Wolfgang Kahl, Vom weiten Schutzbereich zum engen Gewährleistungsgehalt. Zur Kritik einer neuen Richtung der deutschen Grundrechtsdogmatik, in: Staat 43 (2004) 167-202.

10 Näher Christoph Engel, Das legitime Ziel als Element des Übermaßverbots. Gemeinwohl als Frage der Verfassungsdogmatik, in: Brugger, Kirste und Anderheiden (Hrsg.), Gemeinwohl in Deutschland, Europa und der Welt. Baden-Baden (2002) 103-172. 
rechtfertigt Regeln im allgemeinen Polizeirecht, im Umweltrecht oder im Versammlungsrecht, die der Exekutive Ermessen oder wenigstens einen Beurteilungsspielraum einräumen. In den Beispielsfällen ist aber nicht nur das normative Ziel zweifelsfrei. Das Leben ist von Art. 2 Abs. 1 GG geschützt, die Umwelt von Art. 20a GG und der Landfrieden von Art. 8 Abs. 2 GG. Überdies ist auch die Kausalkette zwischen dem Ziel des staatlichen Eingriffs und dem geschützten Rechtsgut kurz. Wenn ein Ziel weniger offensichtlich und wenn die Kausalkette viel länger ist, dann steht das Verfassungsrecht vor einer Entscheidung. Soll es bestimmte Ziele von vornherein ausschließen? Soll die verfassungsrechtliche Rechtfertigung von vornherein ausscheiden, wenn nur das Argument mit einer langen Kausalkette möglich ist ${ }^{11}$ ? Das Bundesverfassungsgericht war mit solchen Überlegungen vor allem bei der Bewältigung der Wiedervereinigung befasst. Der Wunsch der bundesdeutschen Alteigentümer nach der Rückgabe ihrer Grundstücke stieß sich an dem Wunsch der bisherigen Bewohner an der Fortsetzung einer oft langjährigen Nutzung. Das Gericht hat dem Gesetzgeber gestattet, in solchen Fällen zur Herstellung von Rechtsfrieden gestaltend tätig zu werden ${ }^{12}$.

Mich überzeugt das. Die Verfassung sollte besser prozedural reagieren. Dann schlägt sie dem Gesetzgeber eine Begründung zwar nicht von vornherein aus der Hand. Je weniger eindeutig die Entscheidung der Verfassung für das normative Anliegen ist, und je länger die behauptete Kausalkette, desto besser muss aber der Nachweis sein, auf den der Gesetzgeber sich stützen kann. Diese zweite Position vermittelt offensichtlich weniger Rechtssicherheit. Trotzdem glaube ich, dass sich das Verfassungsrecht mit kategorischen Verboten zurückhalten sollte. Man kann ganz konkrete politische Entscheidungen dem Zugriff des Gesetzgebers entziehen. Aber das Verfassungsrecht verhebt sich, wenn es dem Gesetzgeber ganze Klassen von Begründungssträngen verbieten will.

\section{Das normative Gewicht einer Erosion der Einstellung zur Marktwirtschaft}

Das Grundgesetz hat sich jedenfalls nicht explizit für die Marktwirtschaft entschieden ${ }^{13}$. Hätte die Sorge des Gesetzgebers um die Erosion der Zustimmung zur Marktwirtschaft gleichwohl verfassungsrechtliches Gewicht? Tatsächlich ist eine Marktwirtschaft in hohem Maße auf diese Zustimmung angewiesen. Um funktionieren zu können, braucht sie nämlich ein kompliziertes Geflecht an Regeln. Gelegentlich können und müssen diese Regeln mit der souveränen Gewalt des Staates durchgesetzt werden. Das muss aber die seltene Ausnahme bleiben. Wenn die Bürger diese Regeln nicht fast immer ohne weiteres beachten, bricht die Marktwirtschaft zusammen. Je mehr sich die Bürger innerlich von der Marktwirtschaft abwenden, umso mehr wächst diese Gefahr.

11 Dazu neigt mit klugen Gründen Jörn Lüdemann, Edukatorisches Staatshandeln. Baden-Baden (2004).

12 BVerfG 13.3.2001, R 19 - Sachenrechtsmoratorium; BVerfG 16.5.2001, R 15 und R 27 - Ankaufsrecht.

13 Näher BVerfGE 50, 290 - Mitbestimmung. 
Nehmen wir ein einfaches Beispiel. An Märkten werden Güter gehandelt. Der Wettbewerb zwischen Anbietern und Nachfragern sorgt dafür, dass die Güter an den Ort ihrer produktivsten Verwendung wandern. Zugleich veranlasst er die Anbieter dazu, gerade die Güter zu produzieren, nach denen die Nachfrage besonders groß ist. Die Triebkraft dieses Mechanismus ist das Gewinnstreben der Anbieter und der Wunsch der Nachfrager nach mehr Wohlstand. Die Nachfrager könnten diesem Wunsch noch besser entsprechen, wenn sie den Anbietern die Güter einfach wegnehmen, ohne etwas zu bezahlen. Umgekehrt könnten die Anbieter noch mehr Gewinn machen, wenn sie zu Raubrittern mutieren, die den Bürgern ihr Vermögen abnehmen. Die Rechtsordnung verhindert beides, indem sie Eigentumsrechte schafft, zuordnet und verteidigt ${ }^{14}$. Tatsächlich weiß jeder Ladeninhaber aber, wie wenige Diebstähle schließlich aufgeklärt und vor Gericht geahndet werden. Natürlich wäre mehr möglich. Aber der Aufwand für die Verfolgung von Straftaten kann nicht beliebig gesteigert werden. Letztlich ist die Rechtsordnung darauf angewiesen, dass ihre Regeln fast immer ohne Intervention beachtet werden.

Wer die wachsende Spreizung der Einkommen ungerecht findet, der bricht deshalb nicht in das Haus seines Arbeitgebers ein. Er verzichtet auf Diebstahl auch nicht deshalb, weil er eine Theorie über die institutionelle Infrastruktur einer Marktwirtschaft im Kopf hat und die Marktwirtschaft für die beste aller Wirtschaftsordnungen hält. Er käme gar nicht auf die Idee, einen Einbruch zu begehen ${ }^{15}$. Dass er nicht stiehlt, gehört zu der normativen Grundausstattung, die ihm seine Eltern in der Kindheit mitgegeben haben ${ }^{16}$. Wahrscheinlich würde er auch glauben, dass er sich seiner sozialen Anerkennung und seiner Selbstachtung beraubt. Denn Einbrecher gehören an das unterste Ende der sozialen Leiter.

Aber eine Marktwirtschaft braucht mehr als nur die Bereitschaft, auf Diebstähle zu verzichten. Schon die nächste fundamentale Institution einer Marktwirtschaft ist durch soziale Normen viel weniger gut gesichert. In einer Marktwirtschaft werden Güter nicht von einem zentralen Planer zugeteilt. Man einigt sich dezentral mit einem Anbieter auf die Gegenleistung. Oft ist die Leistung im Zeitpunkt des Vertragsschlusses noch nicht verfügbar. Oder der Erwerber kann die Gegenleistung nicht auf einmal erbringen. Dann müssen sich beide Seiten darauf verlassen können, dass der andere die vertragliche Abrede erfüllen wird. Handwerker sind nicht die einzigen, die darüber klagen, dass die Zahlungsmoral verfällt. Natürlich kann man auf die Erfüllung eines Vertrages klagen. Aber wenn die Nichterfüllung nicht mehr der seltene Ausnahmefall ist, dann ist die Rechtsordnung schnell überfordert.

Nahe liegen vor allem Rechtfertigungsstrategien nach dem Motto: die da oben bereichern sich ja auch ständig auf meine Kosten. Warum soll ich dann der Dumme sein, der seine vertraglichen Pflichten erfüllt? Und schon hat man kein Selbstwertproblem mehr damit, der Versicherung einen Schaden zu melden, den es gar nicht gegeben hat; dem Arbeitgeber Geschäftigkeit vorzutäu-

14 Näher Christoph Engel, Die soziale Funktion des Eigentums, in: Depenheuer, Engel und von Danwitz (Hrsg.), Bericht zur Lage des Eigentums. Berlin (2002) 1-107.

15 Das steht schon bei James Fitzjames Stephen, A General View of the Criminal Law of England. London, Cambridge, (1863),99.

16 Näher Christoph Engel, Learning the Law, in: Journal of Institutional Economics 4 (2008) 275-297. 
schen, während man eine private Angelegenheit regelt; dem Finanzamt zu verschweigen, was man nach Dienstschluss privat noch verdient hat. Die Beispiele sind nicht zufällig. Ökonomen würden all das als Fälle von unbeobachtbarem Handeln bezeichnen.

Das ist der Kern des Problems für die Rechtsordnung. Ironischerweise wird sie umso mehr gebraucht, je stärker das generalisierte Vertrauen in die Marktwirtschaft erodiert. Was früher fast immer von selbst geschah, muss nun immer häufiger durch explizite Anwendung und Vollstreckung des Rechts durchgesetzt werden. Das bedeutet nicht nur mehr Arbeit für die Juristen. Vielmehr muss sich die Rechtsordnung immer intensiver mit den Voraussetzungen ihrer gesellschaftlichen Wirkung auseinander setzen. Es genügt immer weniger, Normativität für selbstverständlich zu halten. Fragen, die traditionell allenfalls die Kriminologen interessiert haben, gehen dann auch den normalen Zivil- und Öffentlichrechtler etwas an. Rechtsregeln müssen die Bedingungen ihrer Verwirklichung mit reflektieren. Dabei kann es nicht bloß darum gehen, Vollzugsdefizite $\mathrm{zu}$ identifizieren ${ }^{17}$. Vielmehr braucht die Rechtsordnung empirisch wie theoretisch gut abgesicherte Vorstellungen über die sozialen Prozesse, die für die Befolgung von Rechtsregeln verantwortlich sind. Der Schlüssel zum Verständnis dieser Prozesse ist das generalisierte Vertrauen in die Gerechtigkeit der vom Recht gestützten Wirtschaftsordnung.

\section{Operationalisierung als die Sorge um das generalisierte Vertrauen}

Der größte Vorzug einer Marktwirtschaft ist zugleich ihr größtes Problem: nach dem berühmten Wort von Adam Smith regiert der Markt „mit unsichtbarer Hand““18. Es gibt keinen Masterplan, den man erklären und dem man zustimmen könnte. Alle Ordnung ist prozedural. Die Rechtsordnung schreibt Spielregeln vor, keine Spielergebnisse. Kein zentraler Planer braucht eine unhandhabbare Menge an Informationen zusammenzuführen. Nichts muss gerichtsfest bewiesen werden. Es genügt, dass ein Käufer das Angebot eines Verkäufers annimmt. Kein Dritter muss davon erfahren. Dass man sich auf so viel Dezentralität einlassen soll, ist nicht intuitiv. Das ist das fundamentale Darstellungsproblem der Marktwirtschaft. Es muss immer wieder neu bewältigt werden.

Die Menschen reagieren auf ihre schwer zu verstehende Wirtschaftsordnung in der gleichen Weise, in der sie auch sonst Umgebungen behandeln, denen sie nicht ausweichen können, deren Wirkkräfte aber nicht sofort einsichtig sind. Sie behandeln ihre Einstellung zur Wirtschaftsordnung als ein Problem, das nicht wohldefiniert ist ${ }^{19}$. Im Alltag sind solche Probleme eher die Re-

17 Näher Gerd Winter, Das Vollzugsdefizit im Wasserrecht. Ein Beitrag zur Soziologie des Öffentlichen Rechts. Berlin (1975); Renate Mayntz, Implementation politischer Programme. Empirische Forschungsberichte. Königstein/Ts. (1980); Adrienne Windhoff-Héritier, Politikimplementation. Ziel und Wirklichkeit politischer Entscheidungen. Königstein/Ts. (1980); Gertrude Lübbe-Wolff, Modernisierung des Umweltordnungsrechts. Vollziehbarkeit - Deregulierung - Effizienz. Bonn (1996).

18 Adam Smith, An Inquiry into the Nature and Causes of the Wealth of Nations. London (1776),IV.II.9.18.

19 Näher zu dieser Unterscheidung Gerd Gigerenzer, Peter M. Todd und ABC Research Group, Simple Heuristics that Make us Smart. New York (1999),3-15. 
gel als die Ausnahme. Die Antwort ist Vertrauen: man lässt sich auf eine Situation ein, obwohl man die Risiken nicht vollständig überblickt, die mit dieser Entscheidung verbunden sind ${ }^{20}$. Wer vertraut, ist deshalb nicht dumm. Er vergisst nicht, dass er eine riskante Entscheidung getroffen hat. Er behält sich vor, seine Entscheidung zu revidieren. Hier wird entscheidend, dass die Bürger die Wirtschaftsordnung als ein nicht wohldefiniertes Problem behandeln. Für ihre Einstellung kommt es nicht auf den Nachweis an, dass die Wirtschaftsordnung versagt hat. Es genügt, wenn sie den Eindruck gewinnen, dass ihr Vertrauen enttäuscht worden ist.

Im Kern ist die Marktwirtschaft eine Verfassung des Zusammenlebens in der Gesellschaft. Empirisch behandeln Menschen solche Probleme vornehmlich als Fairnessprobleme ${ }^{21}$. Sie verwenden ziemlich einfache Muster, um zu Urteilen zu kommen. Ein Extrembeispiel belegt, dass Fairness nicht Gleichverteilung von Einkommen oder Vermögen bedeuten muss. Das indische Kastensystem ist nicht trotz, sondern wegen der Dominanz von Fairnessvorstellungen so lange stabil gewesen. Es beruht auf einer mit vielen formellen und informellen Regeln stabilisierten, alternativen Fairnessnorm: Status.

In Fairnessnormen dürfte die eigentliche Bedeutung eines Konzepts liegen, das sich der strikten wissenschaftlichen Durchdringung von vornherein entzogen hat, der „sozialen Marktwirtschaft“. Die Deutschen haben Ludwig Erhard und Oswald Nell-Breuning abgenommen, dass es bei der fremd wirkenden Marktwirtschaft nicht bloß um die Bereicherung der besonders Begabten und Unbekümmerten gehen werde. Sie haben akzeptiert, dass es für die Übernahme von Risiken eine Prämie geben darf, sogar eine ziemlich große Prämie. Aber bei aller formalen Ungleichheit würde es dabei doch ,gerecht zugehen“.

Genau dieser Glaube ist den Bürgern abhanden gekommen. Letztlich kommt es nicht darauf an, ob sie dabei Recht oder Unrecht haben. In diesem Sinne geht es tatsächlich um ein Darstellungsproblem. Aber diesem Darstellungsproblem kann man nicht mit Rhetorik abhelfen. Die Bürger benutzen vielmehr einen Mechanismus, der sogar im Tierreich nachgewiesen worden ist. Mit den wenigen Informationen, die sie wahrnehmen, versetzen sie sich in die Lage ihres sozialen Gegenübers; „mentalising“ nennt die Psychologie den Mechanismus ${ }^{22}$. Und sie bemühen sich dabei weniger um greifbare Ergebnisse (das Problem war ja nicht wohldefiniert), sondern sie versuchen, die Intentionen ihres Gegenübers zu erfassen. Ist die Annahme berechtigt, dass ihr Gegenüber fair handelt? Hier lag die große gemeinsame Leistung von Ludwig Erhard und Oswald Nell-Breuning: dem einen haben die Menschen abgenommen, dass es ihnen schließlich besser gehen werde; dem anderen, dass soziale Unterschiede nichts mit Verachtung, Hochmut oder Mutwilligkeit zu tun haben würden. Unsere Zeit muss neu lernen, was diese Leistung ver-

20 Näher Christoph Engel. Vertrauen - ein Versuch. Preprints aus der Max-Planck-Projektgruppe Recht der Gemeinschaftsgüter Bonn 1999/12 (1999), http://www.coll.mpg.de/pdf_dat/9912.pdf.

21 S. vor allem Jonathan Haidt, The Emotional Dog and Its Rational Tail. A Social Intuitionist Approach to Moral Judgment, in: Psychological Review 108 (2001) 814-834 und weiter Bruno Frey, Not Just for the Money. An Economic Theory of Personal Motivation. Cheltenham (1997); James Konow, Blind Spots: The Effects of Information and Stakes on Fairness Bias and Dispersion, in: Social Justice Research 18 (2005) 349-390.

22 S. nur Peter Fonagy, György Gergely, Elliot L. Jurist und Mary Target, Affect Regulation, Mentalization and the Development of the Self. New York (2002). 
langt. Und dabei wird es nicht nur um Sonntagsreden gehen, sondern um Taten, denen die Bürger Vertrauen schenken.

\section{Grenzen für Managergehälter als zulässige Reaktion?}

Auf beiden Seiten des Atlantik wird ein gesetzliches Instrument zur Wiederherstellung des generalisierten Vertrauens in die Marktwirtschaft besonders prominent diskutiert: gesetzliche Grenzen für Managergehälter. Sie gelten als das sichtbarste Symbol dafür, dass der Marktwirtschaft ihre solidarische Komponente abhanden gekommen ist.

Der deutsche Gesetzgeber hat sich dazu bislang nicht entschlossen. Er hat aber bereits im Jahre 2005 ein Gesetz verabschiedet, das börsennotierte Unternehmen verpflichtet, die Vorstandsgehälter offenzulegen ${ }^{23}$. Im Jahre 2009 hat der Bundestag ein Gesetz angenommen, das das Verfahren zur Entscheidung über Vorstandsgehälter regelt und dem Aufsichtsrat Kriterien für die Entscheidung an die Hand gibt ${ }^{24}$. Manche Parlamentarier hätten lieber eine klare Höchstgrenze ins Gesetz geschrieben. Dafür hat sich aber keine Mehrheit gefunden.

Nehmen wir um der Klarheit des Arguments willen an, das Gesetz hätte festgeschrieben, dass das Jahreseinkommen, einschließlich des Börsenwerts von Aktienoptionen, 1 Mio $€$ nicht übersteigen darf. Könnten die betroffenen Unternehmen gegen diese Regel mit Aussicht auf Erfolg das Bundesverfassungsgericht anrufen? Wie bei allen wirtschaftsbezogenen Fragen ist die Bestimmung des Schutzbereichs der einschlägigen Grundrechte nicht ganz einfach. Das Gesetz verbietet Anstellungsverträge mit einem höheren Entgelt. Das dürfte einen Eingriff in die von Art. 2 Abs. 1 GG mitgeschützte Vertragsfreiheit des Unternehmens und des Managers darstel$\operatorname{len}^{25}$. Der Manager verliert die Möglichkeit, für außerordentliche Leistung außerordentlich entlohnt zu werden. Das könnte für einen Eingriff in die von Art. 12 Abs. 1 GG geschützte Berufsausübungsfreiheit genügen. Das Unternehmen verliert die Möglichkeit, im Wettbewerb um die beste Organisationsform vor allem auf besonders herausragende Managerpersönlichkeiten zu setzen. Auf mittlere Frist mögen es solche Personen reizvoller finden, in den ungeregelten Bereich der freiberuflichen Beratungsunternehmen zu wechseln. Dieser Verlust an Gestaltungsfreiheit mag für einen Eingriff in die von Art. 9 Abs. 1 GG geschützte Organisationsfreiheit von Gesellschaften genügen. Jedenfalls greifen die in dem schon verabschiedeten Gesetz enthaltenen engeren Regeln für die Ausgestaltung der Arbeit im Aufsichtsrat in diese Freiheit ein. Schließlich könnte man an einen Eingriff in Art. 14 Abs. 1 GG denken. Wenn die Kapitalmärkte der Überzeugung sind, dass die Obergrenze für Managergehälter die Gewinnchancen der Unternehmen schmälert, dann sinkt der Unternehmenswert. Nach weit verbreiteter Ansicht ist er allerdings ohnehin nicht von Art. 14 Abs. 1 GG geschützt ${ }^{26}$.

23 BGBl. 2005 I 2267.

24 BGBl. 2009 I 2509.

25 Näher Wolfram Höfling, Vertragsfreiheit. Ene grundrechtsdogmatische Studie. Heidelberg (1991).

26 Näher Christoph Engel, Eigentumsschutz für Unternehmen, in: Archiv des öffentlichen Rechts 118 (1993) 169-236. 
Wie fast immer wird es schließlich aber weniger auf den Tatbestand als auf die Schranken der Grundrechte ankommen. Im Wege praktischer Konkordanz lässt sich letztlich jede benannte Schranke eines Grundrechts erweitern. Deshalb sollte man nicht zu viel Kraft auf die Frage verwenden, was denn nun zu den „Rechten anderer“ iSv Art. 2 Abs. 1 GG gehört, oder zum „Wohl der Allgemeinheit“ iSv Art. 14 Abs. 2 Satz 2 GG. Kritischer ist die folgende Frage: könnte der Gesetzgeber nur vorbringen, dass er die Ungleichheit der Einkommen vermindern möchte? Oder könnte er im Lichte des oben entwickelten Arguments auch sagen: die Spitzengehälter der Manager sind ein besonders sichtbares Signal dafür, wie gerecht es in einer Marktwirtschaft zugeht. Es spricht viel dafür, dass viele Mitglieder der Bevölkerung dieses Signal verwerten, wenn sie entscheiden, ob sie der Marktwirtschaft weiter vertrauen sollen.

Scheinbar ist das eine rein akademische Frage. Denn jedenfalls würde die Ungleichheit ja vermindert. Warum sollte sich der Gesetzgeber oder das Bundesverfassungsgericht deshalb auf das glatte Eis der Argumentation mit den Einstellungen der Bürger begeben? Die Antwort liegt im Verhältnismäßigkeitsgrundsatz. Dass eine Maßnahme geeignet ist, genügt für ihre Rechtfertigung nicht. Sie muss auch erforderlich und angemessen sein. Hier würde es sofort schwierig. Es käme darauf an, welche Freiheit der Gesetzgeber hat, sein normatives Ziel zu operationalisieren. Diese Freiheit kann nicht vollständig sein. Denn sonst liefe der Maßstab der Erforderlichkeit leer. Eine gleich geeignete, aber weniger belastende Maßnahme kann es nur dann geben, wenn das normative Ziel hinreichend abstrakt bestimmt ist. Nehmen wir an, der Gesetzgeber dürfte nur mehr Gleichheit erzwingen. Dann gäbe es offensichtlich eine Fülle alternativer Möglichkeiten. Insbesondere käme aber zum Tragen, dass die Zahl der Manager mit Einkommen oberhalb von 1 Mio $€$ sehr klein ist. Die betroffenen Unternehmen und Manager könnten deshalb einwenden, dass die Maßnahme für das eigentliche Ziel nur wenig erreicht, ihre Freiheiten aber erheblich beschränkt.

\section{Der empirische Zusammenhang zwischen der Einstellung zur Marktwirtschaft und wahrgenommener Ungleichheit der Einkommen}

Oben habe ich herausgearbeitet, warum der Gesetzgeber grundsätzlich auch handeln darf, um die Einstellung der Bürger zur Marktwirtschaft zu verbessern. Hätte das erörterte Gesetz aber überhaupt diese Wirkung? Genauer: wäre die Erwartung des Gesetzgebers so gut abgesichert, dass er handeln darf, obwohl das normative Anliegen nicht die höchste verfassungsrechtliche Dignität genießt, und obwohl die behauptete Kausalkette von der Entlohnung der Manager zur selbstverständlichen Achtung der institutionellen Rahmenbedingungen einer Marktwirtschaft vorraussetzungsvoll ist?

Für das letzte Glied dieser Kette kann ich keine empirische Evidenz anbieten. Dafür müsste man eine eigene empirische Erhebung anstellen. Das Allensbach Institut könnte zum Beispiel eine Reihe von öffentlich diskutierten Argumenten gegen die Marktwirtschaft auflisten und seine Interviewpartner fragen, wie sehr sie diesen Argumenten zustimmen. Am stärksten wäre die Evi- 
denz, wenn sich zeigte, dass sehr hohe Gehälter für Manager als ganz besonders ungerecht empfunden würden.

Auch aus öffentlich zugänglichen Informationen kann man aber ein relativ deutliches Indiz entnehmen. Dieses Indiz möchte ich im folgenden vorführen. Es gibt einen statistisch signifikanten Zusammenhang zwischen der Einstellung zur Marktwirtschaft und zwei relativ gut messbaren Kennzahlen: der Arbeitslosenquote und dem Ausmaß der Ungleichheit von Einkommen. Genauer: Ungleichheit bleibt auch dann noch eine signifikante Erklärung, wenn man für die alternative Erklärung Arbeitslosigkeit „kontrolliert“. Die Bürger lassen sich bei der Bildung ihrer Einstellung zur Marktwirtschaft also auch maßgeblich von der Größe leiten, für die Gehälter der Spitzenmanager ein Signal sind. Für das Verfassungsrecht ist diese Erkenntnis deshalb wichtig, weil der Gesetzgeber auf das Ausmaß der Arbeitslosigkeit kurzfristig nur sehr viel schwerer Einfluss nehmen kann. Verfassungsrechtlich gesprochen wären Maßnahmen, die an der Arbeitslosigkeit ansetzen, deshalb nicht gleich geeignet.

Zunächst zur Bedeutung von Arbeitslosigkeit. Man sieht mit bloßem Auge, dass es in Abbildung einen engen Zusammenhang gibt zwischen der Höhe der Balken und dem Verlauf der Linie mit den verbundenen Dreiecken. Die Balken tragen die Höhe der Arbeitslosigkeit $a b^{27}$. Die Linie mit den Dreiecken wird erneut von Antworten auf die erste Frage des Instituts für Demoskopie in Allensbach gebildet. Die abhängige Variable ist also die Zustimmung zur Marktwirtschaft. Dieses Mal verwende ich nur die Zahlen für die gesamte Bundesrepublik.

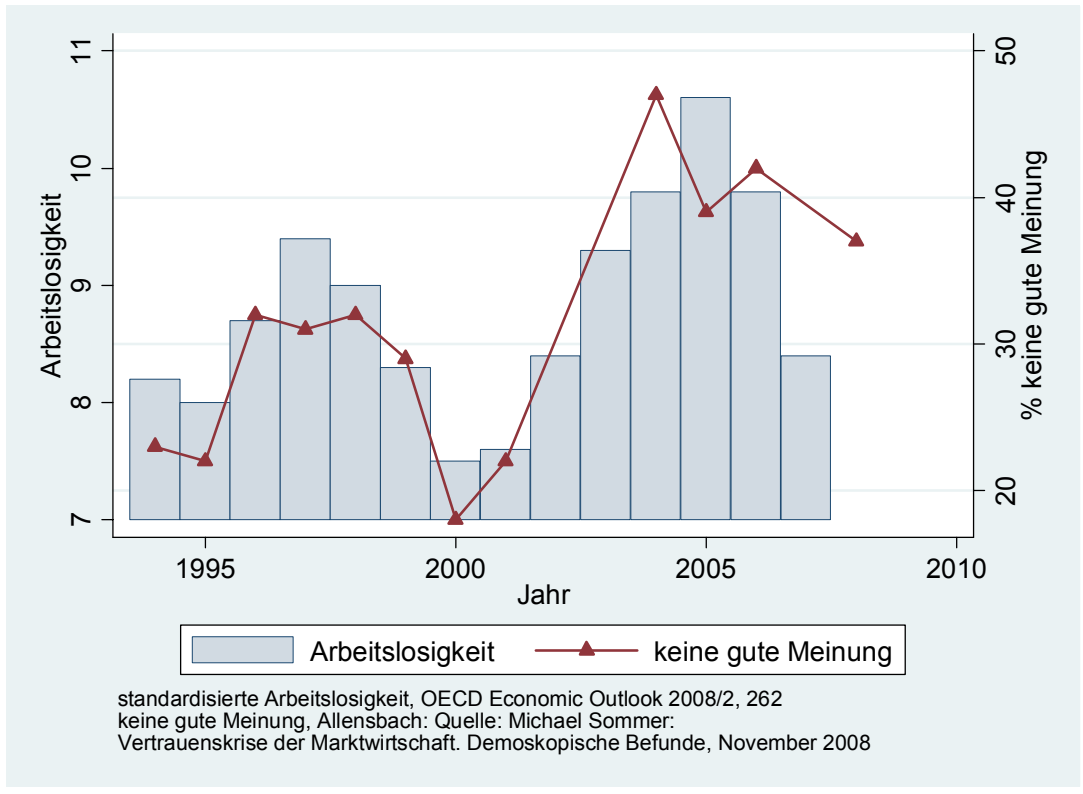

Abbildung 3

Einstellung zur Marktwirtschaft vs. Arbeitslosigkeit

27 Ich verwende die Werte der OECD, weil sie als weniger politisch gefärbt gelten als die parallelen Zahlen der Bundesanstalt für Arbeit. Wenn man deren Zahlen verwendet, ergibt sich aber ein sehr ähnliches Bild. Weil die OECD keine getrennten Zahlen für die alten und die neuen Bundesländer anbietet, verwendet die folgende Grafik im übrigen die Zahlen der BfA. 
Obwohl der ganze Datensatz nur aus 11 Datenpunkten besteht, lässt sich der optische Eindruck statistisch absichern. Der Effekt der Arbeitslosigkeit auf die Zustimmung zur Marktwirtschaft ist hoch signifikant $(\mathrm{p}=.001, \mathrm{t}=4.93)$. Mit einer einzigen erklärenden Variablen (und einer Konstanten) erklärt man $80 \%$ der Varianz ${ }^{28}$. Mit sehr viel größeren Datensätzen erklärt man sehr oft viel weniger.

Zwischen der Einstellung zur Gerechtigkeit der Marktwirtschaft und der Arbeitslosigkeit scheint es keinen ähnlich direkten Zusammenhang zu geben. Zumindest findet man in der Regression in Tabelle 1, Modell 1 aber einen Zusammenhang in der erwarteten Richtung: je höher die Arbeitslosigkeit, desto geringer das Urteil über die Gerechtigkeit der Marktwirtschaft. Dieser Zusammenhang ist aber nur schwach signifikant $(\mathrm{p}=.074)$. Bei nur 10 Datenpunkten kann man das trotzdem berichten. Aber man wird auf die Suche nach alternativen, kraftvolleren Erklärungen gehen.

\begin{tabular}{|l|l|l|l|l|l|}
\hline & Modell 1 & Modell 2 & Modell 3 & Modell 4 & Modell 5 \\
\hline $\begin{array}{l}\text { Arbeitslosigkeit } \\
\text { (OECD) }\end{array}$ & $\begin{array}{l}-3.470^{+} \\
(-2.02)\end{array}$ & & & & $\begin{array}{l}-275.768^{* * *} \\
(-5.40)\end{array}$ \\
\hline Gini YM Becker Hauser & & $\begin{array}{l}-483.259^{* *} \\
(-3.31)\end{array}$ & & & \\
\hline Gini YN Becker Hauser & & & $\begin{array}{l}1734.407^{* *} \\
(5.01)\end{array}$ & & \\
\hline $\begin{array}{l}\text { Gini YM Armutsbericht } \\
\text { (extrapoliert) }\end{array}$ & & & & $\begin{array}{l}-580.995^{* *} \\
(-3.21)\end{array}$ & $\begin{array}{l}-5869.826^{* * *} \\
(-6.05)\end{array}$ \\
\hline $\begin{array}{l}\text { Arbeitslosigkeit* } \\
\text { Gini Armutsbericht }\end{array}$ & & & & & $\begin{array}{l}619.692^{* * *} \\
(5.50)\end{array}$ \\
\hline Konstante & $\begin{array}{l}57.731^{* *} \\
(3.73)\end{array}$ & $\begin{array}{l}251.553^{* *} \\
(3.69)\end{array}$ & $\begin{array}{l}-428.954^{* *} \\
(-4.67)\end{array}$ & $\begin{array}{l}282.680^{* *} \\
(3.54)\end{array}$ & $\begin{array}{l}2633.289^{* * *} \\
(6.00)\end{array}$ \\
\hline $\mathrm{N}$ & 10 & 11 & 11 & 11 & 10 \\
\hline adj.R $\mathrm{R}^{2}$ & .1810 & .4912 & .3494 & .5036 & .7582 \\
\hline p Modell & .0740 & .0079 & .0005 & .0094 & $<.0001$ \\
\hline
\end{tabular}

Tabelle 1

Erklärung der Einstellung zur Gerechtigkeit des Wirtschaftssystems

OLS, White robuste Standardfehler, geclustert für Jahre

t Werte in Klammern, ${ }^{* * *} p<.001,{ }^{* *} p<.01,{ }^{*} p<.05,{ }^{+} p<.1$

28 Technischer: das $\mathrm{R}^{2}$ ist .8022; OLS, White robuste Standardfehler, geclustert für Jahre. Abhängige Variable: Prozent der Befragten, die die Marktwirtschaft im jeweiligen Jahr nicht für gerecht halten. Unabhängige Variable: Maß der OECD für Arbeitslosigkeit, Koeffizient: 8.200. Konstante: $-41.596, p=.013$. Signifikanz des Modells $\mathrm{p}=.0006$. Alle 11 Datenpaare beobachten denselben Gegenstand (die wirtschaftlichen Verhältnisse in Deutschland) zum selben Zeitpunkt. An sich müsste man für diese Abhängigkeit kontrollieren. Hätte man mehrere Zeitreihen (z.B. für verschiedene Länder), dann wäre man mit einem Regressionsmodell jedenfalls auf der sicheren Seite, das für jeden Beobachtungsgegenstand einen fixed effect schätzt. Da wir nur einen einzigen Beobachtungsgegenstand haben, ist das jedoch entbehrlich. 
Das Allensbach Institut hatte seine Frage ja ausdrücklich zugespitzt (,,ich meine, was die Menschen besitzen und was sie verdienen“). Deshalb liegt nahe, dass die Befragten auch von der Gleichheit der Einkommen beeinflusst waren, also gerade von der Größe, auf die ein Gesetz zur Begrenzung der Managergehälter zielen würde. Für Gleichheit gibt es ein eingeführtes quantitatives Maß, den Gini Koeffizienten ${ }^{29}$. Abbildung 4 zeigt, wie das Maß berechnet wird. Man teilt die Bevölkerung nach der Höhe ihres Einkommens oder Vermögens in Gruppen ein. In dem fiktiven Beispiel gibt es fünf Gruppen, sogenannte Quintile. Vollständige Gleichheit wäre erreicht, wenn die untersten 20\% der Bevölkerung genau 20\% des Einkommens erzielen, usw. Vollständige Gleichheit ist also durch die gerade Linie beschrieben. Vollständige Ungleichheit würde bedeuten, dass das gesamte Einkommen oder Vermögen von dem obersten Quintil erzielt wird. Dann gäbe es nur einen Punkt an der oberen rechten Ecke des Diagramms. Empirisch sehen die Verhältnisse häufig so aus wie in dem Beispiel. Die unteren Bevölkerungsgruppen haben einen relativ kleineren, die oberen einen relativ größeren Anteil am Einkommen oder Vermögen. Wie stark die Verteilung von vollständiger Gleichheit abweicht, ergibt sich aus einem Vergleich zwischen der dunklen Fläche und dem Dreieck unter der Geraden. Diese Verhältniszahl ist der Gini Koeffizient.

\section{Gini Koeffizient}

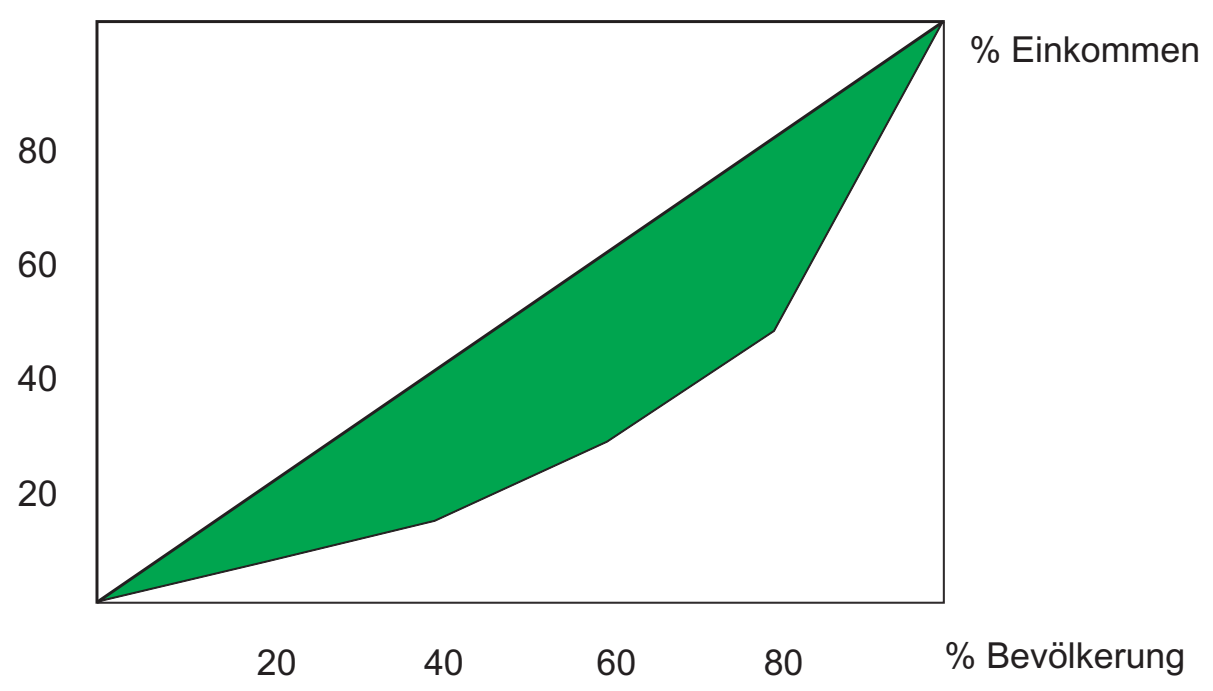

Abbildung 4

Gini Koeffizient

Bei der Anwendung dieses Maßes gibt es zwei Schwierigkeiten. Am liebsten hätte man natürlich den Gini Koeffizienten für jedes Jahr. Tatsächlich sind aber nur Zahlen für bestimmte Stichjahre verfügbar. Deshalb muss man den Koeffizienten für die übrigen Jahre extrapolieren. In den Regressionen in Tabelle 1 geschieht das auf die einfachst denkbare Weise. Der erste verfügbare Wert wird auch für alle vergangenen Jahre angesetzt. Für fehlende Jahre in der Reihe wird der zurückliegende Wert verwendet. 
Die zweite Schwierigkeit ergibt sich aus der Wahl des Gegenstands, dessen Gleichverteilung man betrachtet. Man kann gleichsam brutto oder netto rechnen. In die Berechnung des sogenannten Markteinkommens YM gehen das Einkommen aus unselbstständiger und selbstständiger Arbeit ein, sowie das Einkommen aus Vermögen, einschließlich des Mietwerts selbstgenutzten Eigentums. Das sogenannte Nettoäquivalenzeinkommen YN ist in mehrfacher Hinsicht bereinigt. Ausgangspunkt ist das Markteinkommen. Dazu werden staatliche und private Transfers addiert. Steuern, Pflichtbeiträge zu Sozialversicherungen und freiwillige Aufwendungen für die Krankenversorgung werden abgezogen. Schließlich wird das Ergebnis für die Zusammensetzung des Haushalts gewichtet. Je mehr Personen von dem Einkommen leben müssen, desto geringer das Nettoäquivalenzeinkommen. Haushaltsangehörige, die selbst kein Einkommen erzielen, werden aber nur mit einem Bruchteil angesetzt ${ }^{30}$.

Im Vergleich zwischen den Modellen 2 und 3 in Tabelle 1 sieht man, dass die Menschen offensichtlich keine aufwändigen Verrechnungen vornehmen ${ }^{31}$. Wenn man nur die t Werte der Koeffizienten vergleicht, schneidet der Gini Koeffizient des Nettoäquivalenzeinkommens zwar sogar besser ab. Aber das Modell mit dem Gini Koeffizienten des Markteinkommens erklärt 15\% mehr Varianz. Und vor allem weist das Vorzeichen des Koeffizienten im Modell 3 in eine kontraintuitive Richtung: je höher die Ungleichheit, desto höher die wahrgenommene Gerechtigkeit. Das ist offensichtlich unplausibel. Die Erklärungskraft nimmt noch einmal etwas zu, wenn man im Modell 4 die (extrapolierten) Zahlen aus dem jüngsten Armuts- und Reichtumsbericht der Bundesregierung verwendet ${ }^{32}$. Wirklich gut ist aber erst Modell 5. Es erklärt die wahrgenommene Gerechtigkeit der Wirtschaftsordnung sowohl mit dem Gini Koeffizienten des Markteinkommens wie mit der Arbeitslosigkeit. Zwischen beiden Größen gibt es nahe liegender Weise einen Zusammenhang. Wenn die Arbeitslosigkeit steigt, gibt es mehr Personen mit einem besonders niedrigen Einkommen. Deshalb misst man in Grenzen denselben Effekt zweimal. Der Interaktionseffekt korrigiert diese Verzerrung.

Dieses letzte Modell ist sehr gut. Es erklärt 75\% der Varianz, obwohl insgesamt nur 10 Datenpunkte zur Verfügung stehen. Wie gut das Modell ist, macht die folgende Grafik deutlich. Sie vergleicht die Rohdaten mit der Vorhersage des Modells. Man sieht sofort, dass die Unterschiede sehr klein sind.

30 Einzelheiten bei Silvia Deckl, Indikatoren der Einkommensverteilung in Deutschland 2003. Ergebnisse der Einkommens- und Verbrauchsstichprobe, in: Wirtschaft und Statistik 58 (2006) 1178-1186.

31 Die hier benutzten Gini Koeffizienten sind berechnet von Irene Becker. Entwicklungstendenzen der personellen Einkommensverteilung in Deutschland (2004),

http://www.boeckler.de/pdf/v_makro_2004_10_becker_i.pdf.

BTDrs. 16/9915, 33 . 


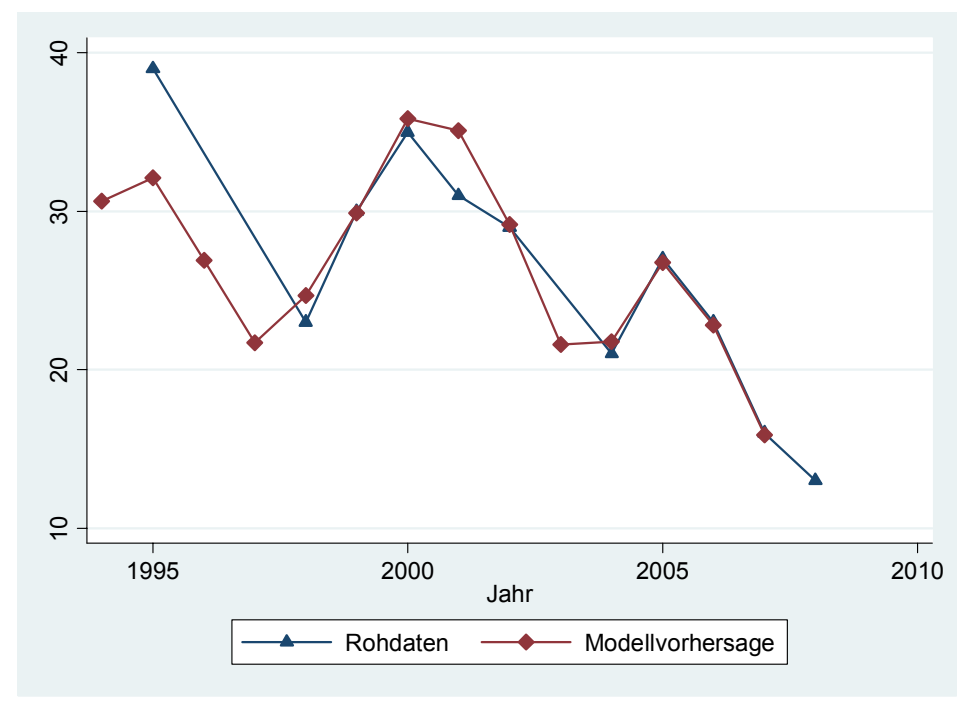

Abbildung 5

Gerechtigkeit: Rohdaten vs. Modellvorhersage

Eigentlich sagt eine Regression nur etwas über einen Zusammenhang. Man kann eine abhängige Variable durch eine oder mehrere unabhängige Variablen mehr oder minder gut erklären. Mehr braucht man auch nicht, wenn man Ereignisse vorhersagen will, die noch gar nicht passiert sind. Für die Rechtspolitik wüsste man jedoch gern mehr. Man möchte Ursache und Wirkung auseinander halten. An sich ist das die Achillesferse der quantitativen Analyse. Ökonometriker werden nicht müde zu betonen, dass eine einfache Regression im Normalfall nicht genügt, um das Identifikationsproblem zu bewältigen ${ }^{33}$. Hat A einen Einfluss auf B gehabt, oder umgekehrt B auf A, oder sind beide Aussagen richtig? Im konkreten Fall braucht uns das aber nicht sonderlich zu beunruhigen. Damit man ein Identifikationsproblem hätte, müsste man behaupten: Menschen werden arbeitslos, weil sie der Marktwirtschaft nicht mehr zustimmen; Menschen erzielen ein niedrigeres Einkommen, weil sie die Marktwirtschaft für ungerechter halten. Beides ist offensichtlich sehr wenig plausibel.

Ein Identifikationsproblem bestünde auch dann, wenn es eine gemeinsame, nicht beobachtete Ursache gäbe, die sowohl die Arbeitslosenquote und den Gini Koeffizienten beeinflusst wie die Einstellung zur Marktwirtschaft. Ein schönes Beispiel für solch ein Problem ist der statistisch signifikante Zusammenhang zwischen der Zahl der Störche und der Zahl der Kinder. Daraus folgt nicht, dass die Störche die Kinder bringen, und auch nicht die Kinder die Störche. Eine plausible Erklärung sind dagegen ländliche Verhältnisse, in denen mehr Mütter zu Hause bleiben. In solchen Umgebungen fühlen sich auch die Störche wohler ${ }^{34}$. Man mag sich fragen, wie sehr die Einstellung zur Marktwirtschaft das Werk von Intermediären ist. Die Medien, die Parteien, die Kirchen und die Verbände mögen sehr wohl dazu beitragen. Ein Identifikationsprob-

33 Eindringlich Edward E. Leamer, Let's Take the Con out of Econometrics, in: American Economic Review 23 (1983) 31-43.

34 Thomas Höfer, Hildegard Pryzrembel und Silvia Verleger, New Evidence for the Theory of the Stork, in: Paedriatric and Perinetal Epidemiology 18 (2004) 88-92. 
lem erwüchse daraus aber erst dann, wenn auch die Arbeitslosigkeit oder die Gleichverteilung der Einkommen maßgeblich von diesen Intermediären bestimmt wäre. Das ist sehr wenig plausibel.

Bleibt die eigentliche Herausforderung. Nehmen wir ein Beispiel: Abbildung 3 zeigt zwar, dass sich die Arbeitslosenquote und das Ausmaß der Zustimmung zur Marktwirtschaft ziemlich genau parallel entwickeln. Aber auch im schwierigsten Jahr, im Jahr 2005, betrug die Arbeitslosigkeit „nur“ 10.6\%. Dagegen hatten 39\% der Befragten dezidiert eine schlechte Meinung von der Marktwirtschaft, und 69\% konnten sich nicht zu der Aussage durchringen, dass sie die Marktwirtschaft für gut halten. Nun mag natürlich manch einer, der selbst Arbeit hat, in seinem engeren Umfeld den Verlust an Einkommen und Entfaltungsmöglichkeiten beobachten, der mit Arbeitslosigkeit einhergeht. Andere mögen um die Verschlechterung der Verhältnisse an den Arbeitsmärkten besorgt sein, auch wenn es sie und ihr Umfeld aller Voraussicht nach gar nicht betreffen wird. Trotzdem bleibt der Unterschied zwischen 10.6\% und 69\% beträchtlich. Mit dem Effekt des Gini-Koeffizienten verhält es sich ähnlich. Die Geschichte würde konsistent, wenn sich die meisten Befragten nicht direkt an den Fundamentaldaten orientieren, sondern an den Signalen, die sie wahrgenommen haben - also auch an den Berichten in den Zeitungen über Managergehälter. Aber ein Beweis ist das natürlich nicht.

Soll sich das Verfassungsrecht auf das Argument trotzdem einlassen? Wie nahezu alle Rechtsfragen ist das letztlich ein Werturteil. Es wäre sicher wünschenswert, dass auch der Zusammenhang zwischen dem Gini Koeffizienten und dem Signal der Managergehälter erst noch in ähnlich belastbarer Weise aufgeklärt würde. Dafür spräche auch, dass demoskopische Umfragen relativ schnell durchgeführt werden können und nicht sehr teuer sind. Wenn das Verfassungsgericht in dem hypothetischen Fall vorsichtiger sein wollte, könnte es die Entscheidung des Gesetzgebers für den konkreten Fall noch akzeptieren, zugleich aber Maßstäbe für das Ausmaß aufstellen, in dem behauptete komplexe Kausalketten aufgeklärt sein müssen. Damit wäre dann nicht nur der Gerechtigkeit im Einzelfall, sondern auch der Fortentwicklung des Verfassungsrechts gedient. 


\section{Preprints 2009}

2009/35: Glöckner A., Kleber J., Tontrup S., Bechtold S., The Endowment Effect in Groups with and without Strategic Incentives

2009/34: Lehmann, S., The German elections in the 1870s: why Germany turned from liberalism to protectionism forthcoming in: Journal of Economic History, In Press.

2009/33: Hakenes H., Schnabel I., Credit Risk Transfer and Bank Competition

2009/32: Jansen J., Beyond the Need to Boast: Cost Concealment Incentives and Exit in Cournot Duopoly

2009/31: Fellner G., Sausgruber R., Traxler C., Testing Enforcement Strategies in the Field: Legal Threat, Moral Appeal and Social Information

2009/30: Lüdemann J., Rechtsetzung und Interdisziplinarität in der Verwaltungsrechtswissenschaft forthcoming in: Öffentliches Recht und Wissenschaftstheorie, Tübingen, Mohr Siebeck, pp. 125-150, In Press.

2009/29: Engel C., Rockenbach B., We Are Not Alone: The Impact of Externalities on Public Good Provision

2009/28: Gizatulina A., Hellwig M., Informational Smallness and the Scope for Limiting Information Rents

2009/27: Hahmeier M., Prices versus Quantities in Electricity Generation

2009/26: Burhop C., The Transfer of Patents in Imperial Germany

2009/25: Burhop C., Lübbers T., The Historical Market for Technology Licenses: Chemicals, Pharmaceuticals, and Electrical Engineering in Imperial Germany

2009/24: Engel C., Competition as a Socially Desirable Dilemma Theory vs. Experimental Evidence

2009/23: Morell A., Glöckner A., Towfigh E., Sticky Rebates: Rollback Rebates Induce Non-Rational Loyalty in Consumers Experimental Evidence

2009/22: Traxler C., Majority Voting and the Welfare Implications of Tax Avoidance

2009/21: Beckenkamp M., Engel C., Glöckner A., Irlenbusch B., Hennig-Schmidt H., Kube S., Kurschilgen M., Morell A., Nicklisch A., Normann H., Towfigh E., Beware of Broken Windows! First Impressions in Public-good Experiments

2009/20: Nikiforakis N., Normann H., Wallace B., Asymmetric Enforcement of Cooperation in a Social Dilemma forthcoming in: Southern Economic Review, In Press.

2009/19: Magen S., Rechtliche und ökonomische Rationalität im Emissionshandelsrecht

2009/18: Broadberry S.N., Burhop C., Real Wages and Labour Productivity in Britain and Germany, 1871-1938: A Unified Approach to the International Comparison of Living Standards

2009/17: Glöckner A., Hodges S.D., Parallel Constraint Satisfaction in Memory-Based Decisions

2009/16: Petersen N., Review Essay: How Rational is International Law?

forthcoming in: European Journal of International Law, vol. 20, In Press.

2009/15: Bierbrauer F., On the legitimacy of coercion for the financing of public goods

2009/14: Feri F., Irlenbusch B., Sutter M., Efficiency Gains from Team-Based Coordination - Large-Scale Experimental Evidence

2009/13: Jansen J., On Competition and the Strategic Management of Intellectual Property in Oligopoly

2009/12: Hellwig M., Utilitarian Mechanism Design for an Excludable Public Good

published in: Economic Theory, vol. 2009, no. July 14, Berlin/Heidelberg, Springer, 2009. 
2009/11: Weinschenk P., Persistence of Monopoly and Research Specialization

2009/10: Horstmann N., Ahlgrimm A., Glöckner A., How Distinct are Intuition and Deliberation? An Eye-Tracking Analysis of Instruction-Induced Decision Modes

2009/09: Lübbers T., Is Cartelisation Profitable? A Case Study of the Rhenish Westphalian Coal Syndicate, 1893-1913

2009/08: Glöckner A., Irlenbusch B., Kube S., Nicklisch A., Normann H., Leading with(out) Sacrifice?

A Public-Goods Experiment with a Super-Additive Player

forthcoming in: Economic Inquiry, In Press.

2009/07: von Weizsäcker C., Asymmetrie der Märkte und Wettbewerbsfreiheit

2009/06: Jansen J., Strategic Information Disclosure and Competition for an Imperfectly Protected Innovation forthcoming in: Journal of Industrial Economics, In Press.

2009/05: Petersen N., Abkehr von der internationalen Gemeinschaft? - Die aktuelle Rechtsprechung des US Supreme Court zur innerstaatlichen Wirkung von völkerrechtlichen Verträgen -

forthcoming in: Völkerrecht im innerstaatlichen Bereich, Vienna, facultas.wuv, In Press.

2009/04: Rincke J., Traxler C., Deterrence Through Word of Mouth

2009/03: Traxler C., Winter J., Survey Evidence on Conditional Norm Enforcement

2009/02: Herbig B., Glöckner A., Experts and Decision Making: First Steps Towards a Unifying Theory of Decision Making in Novices, Intermediates and Experts

2009/01: Beckenkamp M., Environmental dilemmas revisited: structural consequences from the angle of institutional ergonomics, issue 2009/01

\section{Preprints 2008}

2008/49: Glöckner A., Dickert S., Base-rate Respect by Intuition: Approximating Rational Choices in Base-rate Tasks with Multiple Cues

2008/48: Glöckner A., Moritz S., A Fine-grained Analysis of the Jumping to Conclusions Bias in Schizophrenia: DataGathering, Response Confidence, and Information Integration

2008/47: Hellwig M., A Generalization of the Atkinson-Stiglitz (1976) Theorem on the Undesirability of Nonuniform Excise Taxation

2008/46: Burhop C., The Underpricing of Initial Public Offerings in Imperial Germany, 1870-1896

forthcoming in: German Economic Review, In Press.

2008/45: Hellwig M., A Note on Deaton's Theorem on the Undesirability of Nonuniform Excise Taxation forthcoming in: Economics Letters, In Press.

2008/44: Hellwig M., Zur Problematik staatlicher Beschränkungen der Beteiligung und der Einflussnahme von Investoren bei großen Unternehmen

published in: Zeitschrift für das gesamte Handelsrecht und Wirtschaftsrecht, vol. 172, pp. 768-787, 2008.

2008/43: Hellwig M., Systemic Risk in the Financial Sector: An Analysis of the Subprime-Mortgage Financial Crisis published in: De Economist, no. 16.07.2009: Springer US, 2009.

published in: Jelle Zijlstra Lecture, no. 2008/5, Wassenaar, NL, Netherlands Institute for Advanced Study in the Humanities and Social Sciences, Institute of the Royal Netherlands Academy of Arts and Sciences, pp. 100, 2008. 